\title{
Ramsey numbers of sparse hypergraphs
}

\author{
David Conlon* Jacob Fox ${ }^{\dagger} \quad$ Benny Sudakov ${ }^{\ddagger}$
}

\begin{abstract}
We give a short proof that any $k$-uniform hypergraph $H$ on $n$ vertices with bounded degree $\Delta$ has Ramsey number at most $c(\Delta, k) n$, for an appropriate constant $c(\Delta, k)$. This result was recently proved by several authors, but those proofs are all based on applications of the hypergraph regularity method. Here we give a much simpler, self-contained proof which uses new techniques developed recently by the authors together with an argument of Kostochka and Rödl. Moreover, our method demonstrates that, for $k \geq 4$,

$$
c(\Delta, k) \leq 2^{2^{\cdot \cdot 2^{c \Delta}}},
$$

where the tower is of height $k$ and the constant $c$ depends on $k$. It significantly improves on the Ackermann-type upper bound that arises from the regularity proofs, and we present a construction which shows that, at least in certain cases, this bound is not far from best possible. Our methods also allows us to prove quite sharp results on the Ramsey number of hypergraphs with at most $m$ edges.
\end{abstract}

\section{Introduction}

For a graph $H$, the Ramsey number $r(H)$ is the least positive integer $N$ such that, in every twocolouring of the edges of complete graph $K_{N}$ on $N$ vertices, there is a monochromatic copy of $H$. Ramsey's theorem states that $r(H)$ exists for every graph $H$. A classical result of Erdős and Szekeres, which is a quantitative version of Ramsey's theorem, implies that $r\left(K_{k}\right) \leq 2^{2 k}$ for every positive integer $k$. Erdös showed using probabilistic arguments that $r\left(K_{k}\right)>2^{k / 2}$ for $k>2$. Over the last sixty years, there have been several improvements on these bounds (see, e.g., [4]). However, despite efforts by various researchers, the constant factors in the above exponents remain the same.

Determining or estimating Ramsey numbers is one of the central problem in combinatorics, see the book Ramsey theory [17] for details. Besides the complete graph, the next most classical topic in this area concerns the Ramsey numbers of sparse graphs, i.e., graphs with certain upper bound constraints on the degrees of the vertices. The study of these Ramsey numbers was initiated by Burr and Erdös in 1975, and this topic has since placed a central role in graph Ramsey theory. Burr and Erdös conjectured, and it was proved by Chvátal, Rödl, Szemerédi and Trotter [3], that for every graph $G$ on $n$ vertices and maximum degree $\Delta$,

$$
r(G) \leq c(\Delta) n
$$

\footnotetext{
${ }^{*}$ St John's College, Cambridge, United Kingdom. E-mail: D.Conlon@dpmms.cam.ac.uk

${ }^{\dagger}$ Department of Mathematics, Princeton, Princeton, NJ. Email: jacobfox@math.princeton.edu. Research supported by an NSF Graduate Research Fellowship and a Princeton Centennial Fellowship.

${ }^{\ddagger}$ Department of Mathematics, UCLA, Los Angeles, CA 90095 and Institute for Advanced Study, Princeton, NJ. Email: bsudakov@math.ucla.edu. Research supported in part by NSF CAREER award DMS-0546523, NSF grants DMS-0355497 and DMS-0635607, by a USA-Israeli BSF grant, and by the State of New Jersey.
} 
Their proof of this theorem is a classic application of Szemerédi's beautiful regularity lemma. However, the use of this lemma makes the upper bound on $c(\Delta)$ grow as a tower of $2 \mathrm{~s}$ with height proportional to $\Delta$. Eaton [8] used a variant of the regularity lemma to obtain the upper bound $c(\Delta) \leq 2^{2^{c \Delta}}$ for some fixed c. A novel approach of Graham, Rödl, Rucinski [15] that did not use any form of the regularity lemma gives the upper bound $c(\Delta) \leq 2^{c \Delta \log ^{2} \Delta}$ for some fixed $c$. In the other direction, in [16] they proved that there is a positive constant $c$ such that, for every $\Delta \geq 2$ and $n \geq \Delta+1$, there is a bipartite graph $G$ with $n$ vertices and maximum degree at most $\Delta$ satisfying $r(G) \geq 2^{c \Delta} n$. Recently, the authors [5], 12] closed the gap for bipartite graphs by showing that, for every bipartite graph $G$ with $n$ vertices and maximum degree $\Delta, r(G) \leq 2^{c \Delta} n$ for some fixed $c$.

A hypergraph $H=(V, E)$ consists of a vertex set $V$ and an edge set $E$, which is a collection of subsets of $V$. A hypergraph is $k$-uniform if each edge has exactly $k$ vertices. The Ramsey number $r(H)$ of a $k$-uniform hypergraph $H$ is the smallest number $N$ such that, in any 2-colouring of the edges of the complete $k$-uniform hypergraph $K_{N}^{(k)}$, there is guaranteed to be a monochromatic copy of $H$. The existence of these numbers was proven by Ramsey [24, but no proper consideration of the values of these numbers was made until the fifties, when Erdös and Rado [11. To understand the growth of Ramsey numbers for hypergraphs, it is useful to introduce the tower function $t_{i}(x)$, which is defined by $t_{1}(x)=x$ and $t_{i+1}(x)=2^{t_{i}(x)}$, i.e.,

$$
t_{i+1}(x)=2^{2^{\cdot 2^{x}}}
$$

where the number of $2 \mathrm{~s}$ in the tower is $i$. Erdös and Rado showed that for $H$ being the complete $k$-uniform hypergraph $K_{l}^{(k)}, r(H) \leq t_{k}(c l)$, where the constant $c$ depends on $k$. In the other direction, Erdös and Hajnal (see [17]) proved that for $H=K_{l}^{(k)}, r(H) \geq t_{k-1}\left(c l^{2}\right)$, where the constant $c$ depends on $k$.

One can naturally try to extend the sparse graph Ramsey results to hypergraphs. Kostochka and Rödl [20] showed that for every $\epsilon>0$, the Ramsey number of any $k$-uniform hypergraph $H$ with $n$ vertices and maximum degree $\Delta$ satisfies

$$
r(H) \leq c(\Delta, k, \epsilon) n^{1+\epsilon},
$$

where $c(\Delta, k, \epsilon)$ only depends on $\Delta, k$, and $\epsilon$. Since the first proof of the sparse graph Ramsey theorem used Szemerédi's regularity lemma, it was therefore natural to expect that, given the recent advances in developing a hypergraph regularity method [14, 25, 23], linear bounds might as well be provable for hypergraphs. Such a program was indeed recently pursued by several authors (Cooley, Fountoulakis, Kühn, and Osthus [6, 7]; Nagle, Olsen, Rödl, and Schacht [22]; Ishigami [18]), with the result that we now have the following theorem:

Theorem 1 Let $\Delta$ and $k$ be positive integers. Then there exists a constant $c(\Delta, k)$ such that the Ramsey number of any $k$-uniform hypergraph $H$ with $n$ vertices and maximum degree $\Delta$ satisfies

$$
r(H) \leq c(\Delta, k) n
$$

In this paper we will give a short proof of this theorem, which is much simpler and avoids all use of the regularity lemma, building instead on techniques developed recently by Conlon [5] and by Fox and Sudakov [12] in order to study embeddings of sparse bipartite graphs in dense graphs.

The first main result of this paper is an extension of this work from graphs to hypergraphs. An $l$ uniform hypergraph is $l$-partite if there is a partition of the vertex set into $l$ parts such that each edge 
has exactly one vertex in each part. We prove the following Turán-type result for $l$-uniform $l$-partite hypergraphs:

Theorem 2 There exists a constant $c=c(l)$ such that if $F$ is an l-uniform l-partite hypergraph with $n$ vertices and maximum degree $\Delta$ and $G$ is an l-uniform l-partite hypergraph with parts of size $N \geq(\epsilon / 2)^{-c \Delta^{l-1}} n$ and at least $\epsilon N^{l}$ edges, then $G$ contains a copy of $F$.

Then, in section 3, we will prove Theorem 1 by applying an argument of Kostochka and Rödl which shows that the Ramsey problem for general hypergraphs may be reduced to an application of the Turán theorem in the $l$-uniform $l$-partite case. This argument combined with our Theorem 2 shows that, for $k \geq 4$ and $k$-uniform hypergraph $H$ with $n$ vertices and maximum degree $\Delta$,

$$
r(H) \leq t_{k}(c \Delta) n,
$$

where the constant $c$ depends on $k$. For $k=3$, the proof shows that $r(H) \leq t_{3}(c \Delta \log \Delta) n$. This is clearly much better than the Ackermann-type upper bound that arises from the regularity proofs. The tower-type upper bound cannot be avoided as demonstrated by the lower bound of Erdös and Hajnal for the Ramsey number of the complete $k$-uniform hypergraph on $n$ vertices. This hypergraph has maximum degree $\Delta=\left(\begin{array}{l}n-1 \\ k-1\end{array}\right)$ and Ramsey number at least $t_{k-1}\left(c \Delta^{\frac{2}{k-1}}\right) n$, where the constant $c$ depends on $k$.

For $k$-uniform hypergraphs $H_{1}, \ldots, H_{q}$, the multicolour Ramsey number $r\left(H_{1}, \ldots, H_{q}\right)$ is the minimum $N$ such that, in any $q$-colouring of the edges of the complete $k$-uniform hypergraph $K_{N}^{(k)}$ with colours $1, \ldots, q$, there is a monochromatic copy of $H_{i}$ in colour $i$ for some $i, 1 \leq i \leq q$. The proof of Theorem 1 presented here extends in a straightforward manner to the multicolour generalisation, which states that for all positive integers $\Delta, k$, and $q$, there exists a constant $c(\Delta, k, q)$ such that, if $H_{1}, \ldots, H_{q}$ are $k$-uniform hypergraphs each with $n$ vertices and maximum degree $\Delta$, then $r\left(H_{1}, \ldots, H_{q}\right) \leq c(\Delta, k, q) n$. The proof demonstrates that may take $c(\Delta, k, q) \leq t_{k}(c \Delta)$ for $k \geq 4$ and $c(\Delta, 3, q) \leq t_{3}(c \Delta \log \Delta)$, where the constant $c$ depends on $k$ and $q$. In the other direction, in Section 4 we construct, for each sufficiently large $\Delta$, a 3-uniform hypergraph $H$ with maximum degree at most $\Delta$ for which the 4-colour Ramsey number of $H$ satisfies $r(H, H, H, H) \geq t_{3}(c \Delta) n$, where $n$ is the number of vertices of $H$. This example shows that our upper bound for hypergraph Ramsey numbers is probably close to being best possible.

The same example also shows that there is a 3-uniform hypergraph $H$ with $m$ edges for which the 4-colour Ramsey number of $H$ is at least $t_{3}(c \sqrt{m})$. On the other hand, one can easily deduced from the proof of Theorem 1 that for any $k$-uniform hypegraph $H$ with $m$ edges, we have that the $q$-colour Ramsey number of $H$ satisfies $r(H, \cdots, H) \leq t_{3}(c \sqrt{m} \log m)$ for $k=3$, and $r(H, \cdots, H) \leq t_{k}(c \sqrt{m})$ for $k \geq 4$, where $c$ depends on $k$ and $q$.

\section{A Turán theorem for l-uniform l-partite hypergraphs}

The following is a generalisation to hypergraphs of a lemma which has appeared increasingly in the literature on Ramsey theory, whose proof uses a probabilistic argument known as dependent random choice. Early versions of this technique were developed in the papers [13, [19], 26]. Later, variants were discovered and applied to various Ramsey and density-type problems (see, e.g., [21, 1, 27, 20, 12, 5]). We define the weight $w(S)$ of a set $S$ of edges in a hypergraph to be the size of the union of these edges. 
Lemma 1 Suppose $s, \Delta$ are positive integers, $\epsilon, \beta>0$, and $G_{r}=\left(V_{1}, \cdots, V_{r} ; E\right)$ is an $r$-uniform $r$-partite hypergraph with $\left|V_{1}\right|=\left|V_{2}\right|=\cdots=\left|V_{r}\right|=N$ and at least $\epsilon N^{r}$ edges. Then there exists an $(r-1)$-uniform $(r-1)$-partite hypergraph $G_{r-1}$ on the vertex sets $V_{2}, \cdots, V_{r}$ which has at least $\frac{\epsilon^{s}}{2} N^{r-1}$ edges and such that for each nonnegative integer $w \leq(r-1) \Delta$, there are at most $4 r \Delta \epsilon^{-s} \beta^{s} w^{r \Delta} r^{w} N^{w}$ dangerous sets of edges of $G_{r-1}$ with weight $w$, where a set $S$ of edges of $G_{r-1}$ is dangerous if $|S| \leq \Delta$ and the number of vertices $v \in V_{1}$ such that for every edge $e \in S, e+v \in G_{r}$ is less than $\beta N$.

Proof: Let $\mathcal{C}$ be the complete $(r-1)$-uniform $(r-1)$-partite hypergraph on the vertex sets $V_{2}, \cdots, V_{r}$. For any edge $e$ in $\mathcal{C}$, let $d(e)$ be the degree of $e$ in $G_{r}$, i.e., the number of vertices in $V_{1}$ such that $e+v \in G_{r}$. Let $T$ be a set of $s$ random vertices of $V_{1}$, chosen uniformly with repetitions. Let $A$ be the set of edges in $\mathcal{C}$ which are common neighbours of the vertices of $T$, i.e., an edge $e$ of $\mathcal{C}$ is in $A$ if $e+v$ is an edge of $G_{r}$ for all $v \in T$. Let $X$ denote the cardinality of $A$. We will show that with positive probability, the set $A$ will be the edge set of a hypergraph $G_{r-1}$ on vertex sets $V_{2}, \ldots, V_{r}$ with the desired properties. By linearity of expectation and by convexity of $f(z)=z^{s}$,

$$
\begin{aligned}
\mathbb{E}[X] & =\sum_{e \in \mathcal{C}} \mathbb{P}[e \in A]=\sum_{e \in \mathcal{C}}\left(\frac{d(e)}{N}\right)^{s} \\
& \geq \frac{N^{r-1}\left(\frac{\sum_{e \in \mathcal{C}} d(e)}{N^{r-1}}\right)^{s}}{N^{s}} \geq \frac{N^{r-1}(\epsilon N)^{s}}{N^{s}}=\epsilon^{s} N^{r-1} .
\end{aligned}
$$

Note that $X \leq N^{r-1}$ since $\mathcal{C}$ has $N^{r-1}$ edges. Letting $p$ denote the probability that $X \geq \mathbb{E}[X] / 2$, we have

$$
\mathbb{E}[X] \leq(1-p) \mathbb{E}[X] / 2+p N^{r-1} \leq \mathbb{E}[X] / 2+p N^{r-1} .
$$

So the probability $p$ that $X \geq \mathbb{E}[X] / 2 \geq \epsilon^{s} N^{r-1} / 2$ satisfies $p \geq \frac{\mathbb{E}[X]}{2 N^{r-1}} \geq \epsilon^{s} / 2$.

The number of subsets $S$ of $V_{2} \cup \ldots \cup V_{r}$ of size $w$ is $\left(\begin{array}{c}(r-1) N \\ w\end{array}\right)$. For a given $w$-set $S$, the number of collections $\left\{e_{1}, \ldots, e_{t}\right\}$ of size $t$ with $\left|e_{i}\right|=r-1$, and $e_{i} \subset S$ for $1 \leq i \leq t$ is $\left(\begin{array}{c}w \\ r-1 \\ t\end{array}\right)$ ). Hence, summing over all nonnegative $t \leq \Delta$, the number of sets of edges of $\mathcal{C}$ with weight $w$ and size at most $\Delta$ is at most

$$
\sum_{t=0}^{\Delta}\left(\begin{array}{c}
w \\
r-1 \\
t
\end{array}\right)\left(\begin{array}{c}
(r-1) N \\
w
\end{array}\right) \leq w^{r \Delta}(r N)^{w}=w^{r \Delta} r^{w} N^{w},
$$

Let $Y_{w}$ denote the random variable counting the number of dangerous sets $S$ of edges of $G_{r-1}$ with weight $w$. We next give an upper bound on $\mathbb{E}\left[Y_{w}\right]$. For a given set $S$ of edges of $\mathcal{C}$, the probability that $S$ is a subset of edges of $G_{r-1}$ is $\left(\frac{|N(S)|}{N}\right)^{s}$, where $N(S)$ denotes the set of vertices $v \in V_{1}$ with $v+e$ an edge of $G_{r}$ for all $e \in S$. So if $S$ satisfies $N(S)<\beta N$, then the probability that $S$ is a subset of edges of $G_{r-1}$ is less than $\beta^{s}$. By linearity of expectation, we have $\mathbb{E}\left[Y_{w}\right]<\beta^{s} w^{r \Delta} r^{w} N^{w}$.

Let $\alpha=4 r \Delta \epsilon^{-s}$. Since $Y_{w}$ is a nonnegative random variable, Markov's inequality implies that $\mathbb{P}\left(Y_{w} \geq \alpha \mathbb{E}\left[Y_{w}\right]\right) \leq \frac{1}{\alpha}$. Hence, the probability that there is a nonnegative integer $w \leq(r-1) \Delta$ with $Y_{w} \geq \alpha \beta^{s} w^{r \Delta} r^{w} N^{w}$ is at most $r \Delta / \alpha=\epsilon^{s} / 4$. Since the probability that $X \geq \frac{\epsilon^{s}}{2} N^{r-1}$ is at least $\epsilon^{s} / 2$, we can satisfy the conditions of the lemma with probability at least $\epsilon^{s} / 4$.

By simply iterating the previous lemma $l-1$ times, we obtain the following corollary.

Corollary 1 Suppose $s, \Delta$ are positive integers, $\epsilon, \beta>0$, and $G_{l}=\left(V_{1}, \cdots, V_{l} ; E_{l}\right)$ is an l-uniform l-partite hypergraph with $\left|V_{1}\right|=\left|V_{2}\right|=\cdots=\left|V_{l}\right|=N$ and at least $\epsilon N^{l}$ edges. Let $\delta_{l}=\epsilon$ and 
$\delta_{r-1}=\delta_{r}^{s} / 2$ for $2 \leq r \leq l$. Then, for $1 \leq r \leq l-1$, there are $r$-uniform $r$-partite hypergraphs $G_{r}=\left(V_{l-r+1}, \ldots, V_{l}, E_{r}\right)$ with the following properties:

1. $G_{r}$ has at least $\delta_{r} N^{r}$ edges for $1 \leq r \leq l$, and

2. for $2 \leq r \leq l$ and each nonnegative integer $w \leq(r-1) \Delta$, there are at most $4 r \Delta \delta_{r}^{-s} \beta^{s} w^{r \Delta} r^{w} N^{w}$ dangerous sets of $G_{r-1}$ with weight $w$, where a set $S$ of edges of $G_{r-1}$ is dangerous if $|S| \leq \Delta$ and the number of vertices $v \in V_{l-r+1}$ such that for every edge $e \in S, e+v \in G_{r}$ is less than $\beta N$.

This is all the preparation we need before proving our main contribution, Theorem 2. For the proof, we will use Corollary 1 and then show how to embed $F$ into $G$. The latter part is closely related to the many embedding results proven by Fox and Sudakov in [12]. We will actually prove the following more precise version of Theorem 2 :

Theorem 3 Let $l \geq 3, F$ be an l-uniform l-partite hypergraph, on vertex sets $W_{1}, \cdots, W_{l}$, with at most $n$ vertices and maximum degree $\Delta$. Let $G_{l}$ be an l-uniform l-partite graph, on vertex sets $V_{1}, \cdots, V_{l}$ with $\left|V_{1}\right|=\cdots=\left|V_{l}\right|=N$, with at least $\epsilon N^{l}$ edges. Then, provided that $N \geq(\epsilon / 2)^{-(2 l \Delta)^{l-1}} n, G_{l}$ contains a copy of $F$.

Proof: We apply Corollary 1 with $s=2 l \Delta, \delta_{l}=\epsilon, \delta_{i-1}=\delta_{i}^{s} / 2$ for $2 \leq i \leq l$, and $\beta=2(\epsilon / 2)^{(2 l \Delta)^{l-1}}$ to get hypergraphs $G_{l-1}, \ldots, G_{1}$. It is easy to check by induction on $i$ that $\delta_{l-i}=2^{-\left(s^{i}-1\right) /(s-1)} \epsilon^{s^{i}}$, so

$$
\delta_{1}=2^{-\left(s^{l-1}-1\right) /(s-1)} \epsilon^{s^{l-1}} \geq 2(\epsilon / 2)^{(2 l \Delta)^{l-1}}=\beta
$$

and $\delta_{1} N \geq \beta N \geq 2 n$.

We now construct an $l \Delta$-uniform bad hypergraph $B$ with vertex set $V_{1} \cup \ldots \cup V_{l}$ where each edge of $B$ has exactly $\Delta$ vertices in each $V_{i}$. A set $T \subset V_{1} \cup \ldots \cup V_{l}$ which contains exactly $\Delta$ vertices in each $V_{i}$ is an edge of $B$ if and only if there is a dangerous set $S$ of edges of $G_{r}$ for some $r, 1 \leq r \leq l-1$, such that the union of the edges of $S$ is a subset of $T$. In other words, an edge of $B$ is just an extension of the union of the edges of a dangerous set. For a particular dangerous set $S$ of edges with weight $w$ in some $G_{r}$, the number of edges of $B$ that are extensions of the union of the edges in $S$ is at most $N^{l \Delta-w}$ since there are at most $N$ ways to pick each of the $l \Delta-w$ remaining vertices that make up an edge. Summing over all $r$ and $w$, and using the fact that $l \geq 3$ and $\delta_{2}^{s}=2 \delta_{1} \geq 2 \beta$, the number of edges of $B$ is at most

$$
\begin{aligned}
\sum_{r=2}^{l} \sum_{w=0}^{(l-1) \Delta} 4 r \Delta \delta_{r}^{-s} \beta^{s} w^{r \Delta} r^{w} N^{l \Delta} & \leq l^{2} \Delta \cdot 2 l \Delta \beta^{s-1}(l \Delta)^{l \Delta} l^{l \Delta} N^{l \Delta}=2 l^{3} \Delta^{2}\left(l^{2} \Delta\right)^{l \Delta} \beta^{l \Delta-1} \beta^{l \Delta} N^{l \Delta} \\
& \leq 2^{1+3(l \Delta)^{2}} \beta^{l \Delta-1} \beta^{l \Delta} N^{l \Delta} \leq 2^{1+3(l \Delta)^{2}} 2^{\left(1-(2 l \Delta)^{l-1}\right)(l \Delta-1)} \beta^{l \Delta} N^{l \Delta} \\
& \leq 2^{-4(l \Delta)^{2}} \beta^{l \Delta} N^{l \Delta}<\left(\frac{\beta}{4 l \Delta}\right)^{l \Delta}\left(\begin{array}{c}
N \\
l \Delta
\end{array}\right) .
\end{aligned}
$$

Call a set $U \subset V_{1} \cup \ldots \cup V_{l}$ with at most $\Delta$ vertices in each $V_{i}$ bad if there are at least

$$
\left(\frac{\beta}{4 l \Delta}\right)^{l \Delta-|U|}\left(\begin{array}{c}
N \\
l \Delta-|U|
\end{array}\right)
$$

edges of $B$ that contain $U$; otherwise call $U$ good. Note that the above calculation on the number of edges of $B$ demonstrates that the empty set is good. We next prove the following important claim. 
Claim 1 If $S$ is a dangerous set of edges in $G_{r}$ for some $r, 1 \leq r \leq l-1$, and $U$ is a good set, then the union of the edges in $S$ is not a subset of $U$.

Proof: Suppose for contradiction that the union of the edges in $S$ is a subset of $U$. The number of extensions of $U$ to a set which contains exactly $\Delta$ vertices in each $V_{i}$ is

$$
\prod_{i=1}^{l}\left(\begin{array}{l}
N-\left|V_{i} \cap U\right| \\
\Delta-\left|V_{i} \cap U\right|
\end{array}\right)
$$

since we can pick for each $i$ any $\Delta-\left|V_{i} \cap U\right|$ vertices of $V_{i} \backslash U$ to extend $U$. By definition, all of these sets are edges in $B$. Using the simple fact that if $x_{1}, \ldots, x_{l}$ are nonnegative integers then $\prod_{i=1}^{l} x_{i} ! \leq\left(\sum_{i=1}^{l} x_{i}\right)$ !, it is straightforward to check that

$$
\begin{aligned}
\prod_{i=1}^{l}\left(\begin{array}{c}
N-\left|V_{i} \cap U\right| \\
\Delta-\left|V_{i} \cap U\right|
\end{array}\right) & \geq(N / 2)^{l \Delta-|U|} \prod_{i=1}^{l}\left(\Delta-\left|V_{i} \cap U\right|\right) !^{-1} \geq\left(\frac{1}{2}\right)^{l \Delta-|U|}\left(\begin{array}{c}
N \\
l \Delta-|U|
\end{array}\right) \\
& \geq\left(\frac{\beta}{4 l \Delta}\right)^{l \Delta-|U|}\left(\begin{array}{c}
N \\
l \Delta-|U|
\end{array}\right),
\end{aligned}
$$

which contradicts $U$ being good.

Given a good set $U$ with $\left|V_{i} \cap U\right|<\Delta$ and $v \in V_{i} \backslash U$, we say $v$ is bad with respect to $U$ if $U \cup\{v\}$ is bad. Let $B_{U}$ denote the set of vertices that are bad with respect to $U$. We will show that for $U$ good we have $\left|B_{U}\right| \leq \frac{\beta N}{4 l \Delta}$. Indeed, suppose $\left|B_{U}\right|>\frac{\beta N}{4 l \Delta}$. Then the number of edges of $B$ containing $U$ is at least

$$
\frac{\left|B_{U}\right|}{l \Delta-|U|}\left(\frac{\beta}{4 l \Delta}\right)^{l \Delta-|U|-1}\left(\begin{array}{c}
N \\
l \Delta-|U|-1
\end{array}\right)>\left(\frac{\beta}{4 l \Delta}\right)^{l \Delta-|U|}\left(\begin{array}{c}
N \\
l \Delta-|U|
\end{array}\right),
$$

contradicting the fact that $U$ is good.

Fix a labeling $\left\{v_{1}, \cdots, v_{n}\right\}$ of the vertices of $F$ such that all vertices in $W_{i+1}$ precede all those in $W_{i}$ for all $i=1, \cdots, l-1$. For each $i$, let $L_{i}=\left\{v_{1}, \cdots, v_{i}\right\}$. For each vertex $v_{h}$, the trace neighbourhood $N\left(v_{h}\right)$ is the set of vertices $v_{m}$ with $m<h$ that are in an edge of $F$ with $v_{m}$. Note that $N\left(v_{h}\right)$ contains at most $\Delta$ vertices in each $W_{r}$ since $F$ has maximum degree $\Delta$. We will find an embedding $f$ of the vertices of $F$ such that $f\left(W_{r}\right) \subset V_{r}$ for $1 \leq r \leq l$ and for each $i \leq l N$,

1. $f\left(N(v) \cap L_{i}\right)$ is good for each vertex $v$ of $F$, and

2. $f\left(e \cap L_{i}\right)$ is an edge of $G_{r}$ for each edge $e$ of $F$, where $r=\left|e \cap L_{i}\right|$.

The proof will be complete once we find such an embedding $f$ since, for each edge $e$ of $F, f\left(e \cap L_{n}\right)=$ $f(e)$ is an edge of $G_{l}$, so $f$ provides an embedding of $F$ in $G_{l}$. The embedding will be constructed one vertex at a time, in increasing order of subscript, so the proof will be by induction on $i$. As noted earlier, the empty set is good, so our base case $i=0$ is satisfied.

Suppose then that at step $i$, we have found an embedding $f$ of $v_{1}, \ldots, v_{i}$ such that

1. for each vertex $v$ of $F, f\left(N(v) \cap L_{i}\right)$ is good, and

2. for each edge $e$ of $F, f\left(e \cap L_{i}\right)$ is an edge of $G_{r}$, where $r=\left|e \cap L_{i}\right|$. 
Let $j$ be such that $v_{i+1} \in W_{j}$. Let $e_{1}, \ldots, e_{d}$ denote the edges of $F$ that contain $v_{i+1}$ and $e_{1}^{\prime}, \ldots, e_{d}^{\prime}$ denote the truncations of $e_{1}, \ldots, e_{d}$ by deleting all $j$ vertices from each $e_{t}$ that are in some $W_{h}$ with $h \leq j$. Each $e_{t}^{\prime}$ consists of one vertex from each $W_{h}$ with $h>j$. Also, $d \leq \Delta$ since $F$ has maximum degree $\Delta$.

Since $F$ has maximum degree $\Delta$, there are less than $l \Delta$ vertices $v$ for which $v_{i+1} \in N(v)$. For each such $v, f\left(N(v) \cap L_{i}\right)$ is good, so there are at most $\frac{\beta}{4 l \Delta} N$ vertices $w$ in $V_{j}$ for which $f\left(N(v) \cap L_{i}\right) \cup w$ is bad. Adding over all such $v$, we conclude that there are at most $\frac{\beta}{4} N$ bad vertices in all associated with $v_{i+1}$.

Suppose we are still embedding vertices of $W_{l}$ in $V_{l}$. Since the edge set of $G_{1}$ is just a subset of $V_{l}$ whose size by Corollary 1 is at least $\delta_{1} N=\beta N$, then we can choose any of these at least $\beta N$ vertices other than $f\left(v_{1}\right), \ldots, f\left(v_{i}\right)$ for $f\left(v_{i+1}\right)$ to satisfy the second of the two desired properties for $f\left(v_{i+1}\right)$. We see that there are at least $\beta N-i-\frac{\beta}{4} N>\frac{3 \beta N}{4}-n>0$ vertices to choose from for $f\left(v_{i+1}\right)$ to satisfy both of the desired properties.

If, now, we have chosen all of the vertices in $W_{l}, \cdots, W_{j+1}$ and we are trying to embed vertex $v_{i+1}$ in $W_{j}$ (we may have already embedded other vertices in $W_{j}$ ), we can do so. To see this, by the induction hypothesis, $f\left(N\left(v_{i+1}\right) \cap L_{i}\right)=f\left(N\left(v_{i+1}\right)\right)=\bigcup_{t=1}^{d} f\left(e_{t}^{\prime}\right)$ is good. By Claim 1, this implies that the set $\left\{f\left(e_{1}^{\prime}\right), \ldots, f\left(e_{d}^{\prime}\right)\right\}$ of edges of $G_{l-j}$ is not dangerous, i.e., there are at least $\beta N$ vertices $v \in V_{j}$ such that $f\left(e_{t}^{\prime}\right) \cup v$ is an edge of $G_{l-j+1}$ for $1 \leq t \leq d$. Therefore, since there are at most $\frac{\beta}{4} N$ bad vertices associated with $v_{i+1}$ and we have already chosen $f\left(v_{1}\right), \ldots, f\left(v_{i}\right)$, we have at least $\frac{3}{4} \beta N-i>\frac{3}{4} \beta N-n>0$ choices for $f\left(v_{i+1}\right)$, which completes the proof.

\section{The Ramsey theorem}

We are now ready to prove Theorem 1 in the following form:

Theorem 4 Let $\Delta$ and $k \geq 3$ be positive integers. Then the Ramsey number of any $k$-uniform hypergraph $H$ with $n$ vertices and maximum degree $\Delta$ satisfies

$$
r(H) \leq r_{k}(k \Delta)^{\left(2 k \Delta^{2}\right)^{k \Delta}} n
$$

where $r_{k}(l)=r\left(K_{l}^{(k)}\right)$.

Proof: We use the argument of Kostochka and Rödl [20] together with Theorem 3. Let $l=(k-$ 1) $\Delta+1$. Suppose we have a red-blue colouring of the complete $k$-uniform hypergraph on $N$ vertices. Let $G$ be the hypergraph consisting of all the red edges and let $r_{k}(l)$ be the Ramsey number of the hypergraph $K_{l}^{(k)}$. Then, in each subset of the vertices of size $r_{k}(l)$, there is at least one monochromatic $K_{l}^{(k)}$. Counting over all such sets and dividing out by possible multiple counts we see that we have at least

$$
\frac{\left(\begin{array}{c}
N \\
r_{k}(l)
\end{array}\right)}{\left(\begin{array}{c}
N-l \\
r_{k}(l)-l
\end{array}\right)} \geq \frac{N^{l}}{r_{k}(l)^{l}}
$$

monochromatic $K_{l}^{(k)}$. Therefore, either $\mathrm{G}$ or its complement $\bar{G}$ contains at least $N^{l} / 2 r_{k}(l)^{l}$ cliques $K_{l}^{(k)}$. We will suppose that it is $G$. 
Now we pass instead to considering the $l$-uniform hypergraph $G^{(l)}$, the edges of which are exactly those $l$-tuples which form complete $K_{l}^{(k)}$ in $G$. This hypergraph has at least $N^{l} / 2 r_{k}(l)^{l}$ edges. Partition its vertex set randomly into $l$ parts $V_{1}, \cdots, V_{l}$ of equal size $N / l$. The total number of partitions is $\frac{N !}{(N / l) !}$ and, for any given edge $e$, there are $l ! \frac{(N-l) !}{(N / l-1) ! l}$ partitions such that each vertex of this edge is in a different part of the partition. Therefore, the expected number of edges with one vertex in each set of the random partition is at least

$$
e\left(G^{(l)}\right)\left(l ! \frac{(N-l) !}{(N / l-1) ! l} / \frac{N !}{(N / l) !^{l}}\right) \geq \frac{N^{l}}{2 r_{k}(l)^{l}} \cdot l ! \frac{(N-l) !}{(N / l-1) !} \frac{(N / l) !^{l}}{N !} \geq \frac{N^{l}}{2 r_{k}(l)^{l}} \frac{l !}{l^{l}}=\frac{l !}{2 r_{k}(l)^{l}}\left(\frac{N}{l}\right)^{l} .
$$

Now choose such a partition and let $\hat{G}^{(l)}$ be the $l$-uniform $l$-partite subhypergraph of $G^{(l)}$ consisting of those edges of $G^{(l)}$ which have one edge in each partite set. Note that $\hat{G}^{(l)}$ has $N / l$ vertices in each part and at least $\epsilon\left(\frac{N}{l}\right)^{l}$ edges, where $\epsilon=\frac{l !}{2 r_{k}(l)^{l}}$.

Now we extend hypergraph $H$ to an $l$-uniform $l$-partite hypergraph $H^{(l)}$. We first note that the vertices of $H$ can be partitioned into $l$ subsets $A_{1}, \ldots, A_{l}$ such that each edge of $H$ has at most one vertex in each part. This is equivalent to saying that the graph $H^{\prime}$ with the same vertex set as $H$ and with two vertices adjacent if they lie in an edge of $H$ has chromatic number at most $l$. Since $H^{\prime}$ has maximum degree at most $(k-1) \Delta$, it has chromatic number at most $(k-1) \Delta+1=l$. For each edge $e$ of $H$, we add one auxiliary vertex to each $A_{i}$ which is disjoint from $e$ (in total $l-k$ vertices). Note that the maximum degree of $H^{(l)}$ remains $\Delta$. The total number of auxiliary vertices added is at most $\frac{\Delta n}{k} \cdot(l-k)<\Delta(\Delta-1) n$ since there are $l-k$ auxiliary vertices for each edge and the total number of edges of $H$ is at most $\frac{\Delta n}{k}$. Hence, $H^{(l)}$ has less than $\Delta^{2} n$ vertices.

Applying Theorem 3 with $F=H^{(l)}, G_{l}=\hat{G}^{(l)}$, and $\epsilon=\frac{l !}{2 r_{k}(l)^{l}}$ we see that, provided

$$
\frac{N}{l} \geq(\epsilon / 2)^{-(2 l \Delta)^{l-1}} \cdot \Delta^{2} n
$$

$\hat{G}^{(l)}$ contains a copy of $H^{(l)}$. But now, by the construction of $H^{(l)}$, this implies that every edge in $H$ is contained inside an edge of $\hat{G}^{(l)}$. But $\hat{G}^{(l)}$ was chosen in such a way that every $k$-tuple within any edge of $\hat{G}^{(l)}$ is an edge in $G$. Therefore $G$ contains a copy of $H$, so we are done.

As mentioned in the introduction, the proof of Theorem 1 presented here extends in a straightforward manner to the following multicolour generalisation.

Theorem 5 For all positive integers $\Delta, k$, and $q$, there exists a constant $c(\Delta, k, q)$ such that, if $H_{1}, \ldots, H_{q}$ are $k$-uniform hypergraphs each with $n$ vertices and maximum degree $\Delta$, then

$$
r\left(H_{1}, \ldots, H_{q}\right) \leq c(\Delta, k, q) n .
$$

The only difference in the proof is in Theorem 4 , where we replace $r_{k}(l)$ by $r_{k}(l ; q)$, the $q$-colour Ramsey number for the complete $k$-uniform hypergraph on $l$ vertices. Erdős and Rado [1] showed that $r_{k}(l ; q) \leq t_{k}(c l)$, where the constant $c$ depends on $k$ and $q$. We therefore may take $c(\Delta, k, q) \leq t_{k}(c \Delta)$ for $k \geq 4$ and $c(\Delta, 3, q) \leq t_{3}(c \Delta \log \Delta)$, where the constant $c$ depends on $k$ and $q$.

Remark: The strong chromatic number of a hypergraph $H$ is the minimum number of colors required to colour the vertices of $H$ so that each edge of $H$ has no repeated colour. The proof of Theorem 4 
demonstrates that if $H$ is a $k$-uniform hypergraph with $n$ vertices, maximum degree $\Delta$, and strong chromatic number $l$, then the $q$-colour Ramsey number of $H$ satisfies

$$
r(H, \cdots, H) \leq r_{k}(l ; q)^{(2 l \Delta)^{l}} .
$$

Indeed, in the proof of Theorem 4, we only used the fact that the vertices of $H$ can be partitioned into $l$ parts such that every edge has at most one vertex in each part.

\section{Lower bound construction}

The following theorem demonstrates that our upper bound for hypergraph Ramsey numbers proved in the previous section is in some cases close to best possible.

Theorem 6 There is $c>0$ such that for each sufficiently large $\Delta$, there is a 3-uniform hypergraph $H$ with maximum degree at most $\Delta$ for which the 4-colour Ramsey number of $H$ satisfies

$$
r(H, H, H, H) \geq 2^{2^{c \Delta}} n,
$$

where $n$ is the number of vertices of $H$.

Proof: Our proof uses the same 4-edge-colouring of the complete 3-uniform hypergraph that was constructed by Erdős and Hajnal (see, e.g., [17]). Not only does this colouring have no large monochromatic complete 3-uniform hypergraph, but we show it also does not have any monochromatic copies of a much sparser 3-uniform hypergraph $H$.

Let $n \geq 4$ be even, $m=\left\lceil 2^{n / 4}\right\rceil$, and suppose the edges of the complete graph $K_{m}$ are coloured red or blue in such a way that neither colour contains a monochromatic copy of the graph $K_{n / 2}$. Such an edge-colouring exists by the lower bound of Erdős (see [17]) on the Ramsey number of the complete graph.

Let $V=\left\{v_{1}, \cdots, v_{n}\right\}$ be a set of vertices and let $H$ be the 3-uniform hypergraph on $V$ whose edge set is given by $\left\{v_{i}, v_{i+1}, v_{j}\right\}$ for all $1 \leq i, j \leq n$. (Note that when $i=n$, we consider $i+1$ to be equal to 1.) It is straightforward to check that every vertex in $H$ has degree $\Delta \leq 3 n$.

We are going to define a 4-colouring of the complete 3 -uniform hypergraph on the set

$$
T=\left\{\left(\gamma_{1}, \cdots, \gamma_{m}\right): \gamma_{i}=0 \text { or } 1\right\}
$$

in such a way that there is no monochromatic copy of $H$. Note that then we will be done, since $T$ has size $2^{m} \geq 2^{2^{n / 4}}$ while $H$ has maximum degree $\Delta \leq 3 n$.

To define our colouring, we need some definitions:

If $\epsilon=\left(\gamma_{1}, \cdots, \gamma_{m}\right), \epsilon^{\prime}=\left(\gamma_{1}^{\prime}, \cdots, \gamma_{m}^{\prime}\right)$ and $\epsilon \neq \epsilon^{\prime}$, define

$$
\delta\left(\epsilon, \epsilon^{\prime}\right)=\max \left\{i: \gamma_{i} \neq \gamma_{i}^{\prime}\right\},
$$

that is, $\delta\left(\epsilon, \epsilon^{\prime}\right)$ is the largest coordinate at which they differ. We can now define an ordering on $T$ by

$$
\begin{aligned}
& \epsilon<\epsilon^{\prime} \text { if } \gamma_{i}=0, \gamma_{i}^{\prime}=1, \\
& \epsilon^{\prime}<\epsilon \text { if } \gamma_{i}=1, \gamma_{i}^{\prime}=0 .
\end{aligned}
$$


Another way of looking at this ordering is to assign to each $\epsilon$ the number $b(\epsilon)=\sum_{i=1}^{m} \gamma_{i} 2^{i-1}$. The ordering then says simply that $\epsilon<\epsilon^{\prime}$ iff $b(\epsilon)<b\left(\epsilon^{\prime}\right)$.

It is important to note the following two properties of the function $\delta$ :

(a) if $\epsilon_{1}<\epsilon_{2}<\epsilon_{3}$, then $\delta\left(\epsilon_{1}, \epsilon_{2}\right) \neq \delta\left(\epsilon_{2}, \epsilon_{3}\right)$;

(b) if $\epsilon_{1}<\epsilon_{2}<\cdots<\epsilon_{r}$, then $\delta\left(\epsilon_{1}, \epsilon_{r}\right)=\max _{1 \leq i \leq r-1} \delta\left(\epsilon_{i}, \epsilon_{i+1}\right)$.

Now we are ready to define our colouring of the complete 3-uniform hypergraph $\tau$ on vertex set $T$. To begin, suppose that $\left\{\epsilon_{1}, \epsilon_{2}, \epsilon_{3}\right\}$ with $\epsilon_{1}<\epsilon_{2}<\epsilon_{3}$ is an edge in $\tau$. Write $\delta_{1}=\delta\left(\epsilon_{1}, \epsilon_{2}\right), \delta_{2}=\delta\left(\epsilon_{2}, \epsilon_{3}\right)$. Then we colour as follows:

$$
\begin{aligned}
& C_{1}, \text { if }\left\{\delta_{1}, \delta_{2}\right\} \text { is red and } \delta_{1}<\delta_{2} ; \\
& C_{2}, \text { if }\left\{\delta_{1}, \delta_{2}\right\} \text { is red and } \delta_{1}>\delta_{2} ; \\
& C_{3}, \text { if }\left\{\delta_{1}, \delta_{2}\right\} \text { is blue and } \delta_{1}<\delta_{2} ; \\
& C_{4} \text {, if }\left\{\delta_{1}, \delta_{2}\right\} \text { is blue and } \delta_{1}>\delta_{2} .
\end{aligned}
$$

Now, let $S=\left\{\epsilon_{1}, \cdots, \epsilon_{n}\right\}_{<}$be an ordered $n$-tuple within $\tau$ and suppose that there is a copy of $H$ on $S$ which is coloured by $C_{1}$. Suppose that the natural cycle $\left\{v_{1}, \cdots, v_{n}\right\}$ associated with $H$ occurs as $\left\{\epsilon_{\pi(1)}, \cdots, \epsilon_{\pi(n)}\right\}$ where $\pi$ is a permutation of $1, \cdots, n$. For each $i, 1 \leq i \leq n$, let $\phi(i)=$ $\max (\pi(i), \pi(i+1))$ and $\psi(i)=\min (\pi(i), \pi(i+1))$.

We claim that $\delta_{\phi(i)-1}=\delta\left(\epsilon_{\phi(i)-1}, \epsilon_{\phi(i)}\right)$ must be larger than $\delta_{j}=\delta\left(\epsilon_{j}, \epsilon_{j+1}\right)$ for all $j<\phi(i)-1$. First consider the triple $\left\{\epsilon_{\psi(i)}, \epsilon_{\phi(i)-1}, \epsilon_{\phi(i)}\right\}_{<}$, which is an edge of the copy of $H$ on $S$. The colouring $C_{1}$ implies that

$$
\delta_{\phi(i)-1}=\delta\left(\epsilon_{\phi(i)-1}, \epsilon_{\phi(i)}\right)>\delta\left(\epsilon_{\psi(i)}, \epsilon_{\phi(i)-1}\right)=\max _{\psi(i) \leq j<\phi(i)-1} \delta_{j} .
$$

This proves the claim for $\psi(i) \leq j<\phi(i)-1$. Next consider the triple $\left\{\epsilon_{j}, \epsilon_{\psi(i)}, \epsilon_{\phi(i)}\right\}<$ with $j<\psi(i)$, which is also an edge of the copy of $H$ on $S$. The colouring $C_{1}$ implies that

$$
\delta_{j} \leq \delta\left(\epsilon_{j}, \epsilon_{\psi(i)}\right)<\delta\left(\epsilon_{\psi(i)}, \epsilon_{\phi(i)}\right)=\delta_{\phi(i)-1} .
$$

This proves the claim in the remaining cases $1 \leq j<\phi(i)-1$.

Consider the set $\{\phi(2 i-1)\}_{i=1}^{n / 2}$, which contains $n / 2$ distinct elements since $\phi(i)=\max (\pi(2 i-1), \pi(2 i))$ and these pairs are disjoint. Let $j_{1}, \ldots, j_{n / 2}$ be a permutation of the odd numbers up to $n-1$ such that $\phi\left(j_{1}\right)<\ldots<\phi\left(j_{n / 2}\right)$. By the claim in the previous paragraph, we have $\delta_{\phi\left(j_{1}\right)-1}<\cdots<\delta_{\phi\left(j_{n / 2}\right)-1}$. Consider, for each $r<s$ with $r, s \in\{1, \cdots, n / 2\}$, the triple $\left\{\epsilon_{\psi\left(j_{r}\right)}, \epsilon_{\phi\left(j_{r}\right)}, \epsilon_{\phi\left(j_{s}\right)}\right\}<$, which is an edge of the copy of $H$ on $S$. Since $\psi\left(j_{r}\right)<\phi\left(j_{r}\right)<\phi\left(j_{s}\right)$, by property (b) of function $\delta$ and the claim above, $\delta\left(\epsilon_{\psi\left(j_{r}\right)}, \epsilon_{\phi\left(j_{r}\right)}\right)=\delta_{\phi\left(j_{r}\right)-1}$ and $\delta\left(\epsilon_{\phi\left(j_{r}\right)}, \epsilon_{\phi\left(j_{s}\right)}\right)=\delta_{\phi\left(j_{s}\right)-1}$. Therefore, by the definition of $C_{1}$ we must have that $\left\{\delta_{\phi\left(j_{r}\right)-1}, \delta_{\phi\left(j_{s}\right)-1}\right\}$ is red. Hence we get a clique of size $n / 2$ in our original colouring. But this cannot happen so we have a contradiction. All other cases follow similarly, so we're done.

This result is closely related to another interesting question: what is the maximum of $r(H)$ over all $k$-uniform hypergraphs with $m$ edges (we assume here that the hypergraphs we consider do not have isolated vertices)? For graphs, this question was posed by Erdős and Graham [10] who conjectured that the Ramsey number of a complete graph is at least the Ramsey number of every graph with the same number of edges. As noted by Erdös [9], this conjecture implies that there is a constant $c$ such that for all graphs $G, r(G) \leq 2^{c \sqrt{e(G)}}$. The best result in this direction, proven by Alon, Krivelevich 
and Sudakov [1], is that $r(G) \leq 2^{c \sqrt{e(G)}} \log e(G)$. For hypergraphs, one can naturally ask a question similar to the Erdös-Graham conjecture, i.e, is there a constant $c=c(k)$ such that for every $k$-uniform hypergraph $H, r(H) \leq t_{k}(c \sqrt[k]{e(H)})$ ? The proof of Theorem 6 has the following corollary:

Corollary 2 There is a positive constant $c$ such that for each positive integer $m$, there is a 3uniform hypergraph $H$ with at most $m$ edges such that the 4-colour Ramsey number of $H$ satisfies $r(H, H, H, H) \geq 2^{2^{c \sqrt{m}}}$.

Indeed the 3-uniform hypergraph $H$ constructed in the proof of Theorem 6 has $n$ vertices and less than $n^{2}$ edges, while $r(H, H, H, H) \geq t_{3}(n / 4) \geq t_{3}(\sqrt{e(H)} / 4)$. This corollary demonstrates that the multicolour version of the hypergraph analogue of the Erdös-Graham conjecture is false.

In the other direction, we prove the following theorem:

Theorem 7 The q-colour Ramsey number of any $k$-uniform hypergraph $H$ with $m$ edges satisfies

$$
r(H, \cdots, H) \leq t_{k}(c \sqrt{m})
$$

for $k \geq 4$, and

$$
r(H, \cdots, H) \leq t_{3}(c \sqrt{m} \log m)
$$

for $k=3$, where constant $c$ depends only on $k$ and $q$.

Theorem 7 follows immediately from the remark after the proof of Theorem 4 together with the following lemma.

Lemma 2 Every $k$-uniform hypergraph $H$ with $m$ edges has strong chromatic number at most $k \sqrt{m}$.

Proof: Let $H^{\prime}$ be the graph on the same vertex set as $H$ with two vertices adjacent if they lie in an edge of $H$. The strong chromatic number of $H$ is clearly equal to the chromatic number of $H^{\prime}$. The number $e\left(H^{\prime}\right)$ of edges of $H^{\prime}$ is at most $\left(\begin{array}{l}k \\ 2\end{array}\right) m \leq\left(\begin{array}{c}k \sqrt{m} \\ 2\end{array}\right)$ since each edge of $H$ gives rise to at most $\left(\begin{array}{l}k \\ 2\end{array}\right)$ edges of $H^{\prime}$. To finish the proof, note that the chromatic number $\chi$ of any graph with $t$ edges satisfies $\left(\begin{array}{l}\chi \\ 2\end{array}\right) \leq t$ because in an optimal colouring there should be an edge between any two colour classes.

\section{Conclusion}

Throughout this paper we have aimed for simplicity in the exposition. Accordingly, in proving Theorem 2, we have cut some corners to make the proof as pithy as possible. The resulting constant, $c=(2 k)^{k-1}$, is doubtless far from best possible, but we believe that this loss is outweighed by the resulting brevity of exposition.

As we noted in the introduction, our Theorem 4 implies that, for $k \geq 4$, there exists a constant $c=c(k)$ such that, for any graph $H$ on $n$ vertices with maximum degree $\Delta, r(H) \leq t_{k}(c \Delta) n$, where the constant $c$ depends only on $k$. For $k=3$, however, it only implies that

$$
r(H) \leq 2^{2^{c \Delta \log \Delta}} n,
$$


which could perhaps be improved a little. It is worth noting also that for $k=2$, the best known bound, proved by Graham, Rödl and Ruciński [15] using a very different method is

$$
r(H) \leq 2^{c \Delta \log ^{2} \Delta} n
$$

In light of the situation for higher $k$ as well as the lower bound constructions for $k=2,3$, the following is a natural question:

Problem 1 Can the log factors in the highest exponent of the upper bounds (11) and (2) be removed?

This problem is certainly difficult in the $k=2$ case, but maybe a different extension of the methods of [12] or an appropriate generalisation of the work of Graham, Rödl and Ruciński could resolve the $k=3$ case.

It also seems likely to us that the lower bound for this problem is essentially the same as the upper bound. So we have the following open problem:

Problem 2 Is it true that for all $k$ and $\Delta$ and sufficiently large $n$, there exists a $k$-uniform hypergraph $H$ with maximum degree $\Delta$ and $n$ vertices such that $r(H) \geq t_{k}(c \Delta) n$, where $c>0$ only depends on $k$ ?

Acknowledgement. We would like to thank Jan Hladky for finding several typos in an earlier version of this paper.

\section{References}

[1] N. Alon, M. Krivelevich, B. Sudakov: Turán numbers of bipartite graphs and related Ramsey-type questions, Combin. Probab. Comput. 12 (2003), 477-494.

[2] S.A. Burr, P. Erdős: On the magnitude of generalized Ramsey numbers for graphs, in: Infinite and Finite Combinatorics, vol. 1, Colloq. Math. Soc. János Bolyai 10 (1975) 214-240.

[3] V. Chvatál, V. Rödl, E. Szemerédi, W.T. Trotter Jr.: The Ramsey number of a graph with bounded maximum degree, J. Combin. Theory Ser. B 34 (1983), 239-243.

[4] D. Conlon: A new upper bound for diagonal Ramsey numbers, Annals of Mathematics, to appear.

[5] D. Conlon: Hypergraph Packing and sparse bipartite Ramsey numbers, submitted.

[6] O. Cooley, N. Fountoulakis, D. Kühn, D. Osthus: 3-uniform hypergraphs of bounded degree have linear Ramsey numbers, to appear in J. Combin. Theory Ser. B.

[7] O. Cooley, N. Fountoulakis, D. Kühn, D. Osthus: Embeddings and Ramsey numbers of sparse $k$-uniform hypergraphs, submitted.

[8] N. Eaton: Ramsey numbers for sparse graphs, Discrete Math. 185 (1998), 63-75.

[9] P. Erdős: On some problems in graph theory, combinatorial analysis and combinatorial number theory, Graph Theory and Combinatorics (Cambridge, 1983), Academic Press, London-New York (1984), 1-17. 
[10] P. Erdős, R.L. Graham: On partition theorems for finite graphs, Infinite and Finite Sets (Colloq., Keszthely, 1973: dedicated to P. Erdös on his 60th birthday), Vol. I; Colloq. Math. Soc. János Bolyai, Vol.10, North-Holland, Amsterdam (1975), 515-527.

[11] P. Erdős, R. Rado: Combinatorial theorems on classifications of subsets of a given set, Proc. London Math. Soc. 3 (1952), 417-439.

[12] J. Fox, B. Sudakov: Density theorems for bipartite graphs and related Ramsey-type results, submitted.

[13] W. T. Gowers: A new proof of Szemerédi's theorem for arithmetic progressions of length four, Geom. Funct. Analysis 8 (1998), 529-551.

[14] W.T. Gowers: Hypergraph regularity and the multidimensional Szemerédi theorem, to appear in Annals of Math.

[15] R.L. Graham, V. Rödl, A. Ruciński: On graphs with linear Ramsey numbers, J. Graph Theory 35 (2000), 176-192.

[16] R.L. Graham, V. Rödl, A. Ruciński: On bipartite graphs with linear Ramsey numbers, Combinatorica 21 (2001), 199-209.

[17] R.L. Graham, B.L. Rothschild, J.L. Spencer: Ramsey theory, John Wiley \& Sons (1980).

[18] Y. Ishigami: Linear Ramsey numbers for bounded-degree hypergraphs, preprint.

[19] A. V. Kostochka, V. Rödl: On graphs with small Ramsey numbers, J. Graph Theory 37 (2001), 198-204.

[20] A. V. Kostochka, V. Rödl: On Ramsey numbers of uniform hypergraphs with given maximum degree, J. Combin. Theory Ser. A 113 (2006), 1555-1564.

[21] A. V. Kostochka, B. Sudakov: On Ramsey numbers of sparse graphs, Combin. Probab. Comput. 12 (2003), 627-641.

[22] B. Nagle, S. Olsen, V. Rödl, M. Schacht: On the Ramsey number of sparse 3-graphs, preprint.

[23] B. Nagle, V. Rödl, M. Schacht: The counting lemma for k-uniform hypergraphs, Random Struct. Algorithms 28 (2006), 113-179.

[24] F.P. Ramsey: On a problem of formal logic, Proc. London Math. Soc. Ser. 230 (1930), 264-286.

[25] V. Rödl, J. Skokan: Regularity lemma for k-uniform hypergraphs, Random Struct. Algorithms 25 (2004), 1-42.

[26] B. Sudakov: Few remarks on the Ramsey-Turan-type problems, J. Combinatorial Theory Ser. B 88 (2003), 99-106.

[27] B. Sudakov: Large $K_{r}$-free subgraphs in $K_{s}$-free graphs and some other Ramsey-type problems, Random Structures \& Algorithms 26 (2005), 253-265. 